\section{Strenge Blutdruckeinstellung von Vorteil}

Die SPRINT-Studie spricht dafür, hypertensive Patienten mit hohem Risiko, jedoch ohne Diabetes mellitus, auf einen Blutdruckzielwert unter $120 \mathrm{mmHg}$ systolisch einzustellen.

$\mathrm{D}$ ie arterielle Hypertonie ist der bedeutendste Risikofaktor für kardiovaskuläre Ereignisse. Es konnte überzeugend gezeigt werden, dass eine medikamentöse Behandlung der arteriellen Hypertonie zu einer signifikanten Senkung der Wahrscheinlichkeit für Schlaganfall, Myokardinfarkt und Herzinsuffizienz führen kann.

Die anzustrebenden Zielwerte für den systolischen Blutdruck bei Patienten mit hohem kardiovaskulären Risiko (z. B. Alter $>75$ Jahren, vorbestehende kardiovaskuläre Erkrankung, chronische Niereninsuffizienz), aber ohne Diabetes mellitus, wurden in der vom US-amerikanischen National Heart, Lung, and Blood Institute (NHLBI) geförderten SPRINTStudie erneut untersucht. In der Studie wurden 9.381 Patienten mit einem systo- lischen Blutdruck $>130 \mathrm{mmHg}$ auf Zielwerte von $<120 \mathrm{mmHg}$ (intensive Einstellung) versus $<140 \mathrm{mmHg}$ (Standardeinstellung) randomisiert.

Die Studie wurde nach etwas über drei Jahren aufgrund einer signifikant niedrigeren Rate an schweren kardiovaskulären Ereignissen in der Gruppe mit dem Zielwert $<120 \mathrm{mmHg}$ vorzeitig gestoppt (Ereignisrate 1,65\% versus 2,19\% pro Jahr, Hazard Ratio [HR] 0,75, p < 0,001). Auch die Gesamtsterblichkeit war in der Behandlungsgruppe mit intensiver Blutdruckeinstellung signifikant niedriger (HR 0,73, p=0,003).

Schwerwiegende Nebenwirkungen wie Hypotension, Synkope, Elektrolytentgleisungen und Niereninsuffizienz/ -versagen traten jedoch in der Gruppe mit niedrigem Zielwert häufiger auf.
The SPRINT Research Group. A Randomized Trial of Intensive versus Standard Blood-Pressure Control. N Engl J Med. 2015; 373: 2103-2116.

\section{Kommentar}

Die SPRINT-Studie wird mit hoher Wahrscheinlichkeit Leitlinienrelevanz haben und zu einer Senkung des systolischen Blutdruckzielwerts für Patienten mit hohem kardiovaskulären Risiko führen (120 mmHg), auch wenn eine Gesamtbetrachtung der Ergebnisse unter Berücksichtigung der Nebenwirkungen einer strengen Blutdruckeinstellung noch nicht abgeschlossen ist. Sicher ist jedoch, dass das Erreichen dieses Blutdruckzielwerts eine große Herausforderung ist und eine engere Anbindung hypertensiver Patienten erfordert.

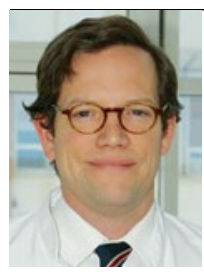

Prof. Dr. Peter W. Radke Klinik für Innere Medizin - Kardiologie, Schön Klinik Neustadt

ten mit prolongierter DAPT. Die Sterblichkeit betrug 2,3\% in der Gruppe mit prolongierter DAPT gegenüber $3,5 \%$ in der ASS-Gruppe (Hazard Ratio 0,65, p = $0,18)$. Schwere Blutungsereignisse traten gleich häufig auf (jeweils 2,0, $\mathrm{p}=0,95$ ).

Helft G et al. Stopping or continuing clopidogrel 12 months after drug-eluting stent placement: the OPTIDUAL randomized trial. Eur Heart J. 2016; 37: 365-374.

\section{Kommentar}

Die Frage nach der optimalen Dauer der dualen Plättchenhemmung nach der Implantation von medikamentenbeschichteten Stents ist weiterhin ungelöst. Für Patienten mit akutem Koronarsyndrom und niedrigem bis moderatem Blutungsrisiko werden die Leitlinien in Zukunft jedoch wahrscheinlich die Möglichkeit einer über zwölf Monate andauernden dualen Plättchenhemmung eröffnen.

Prof. Dr. Peter W. Radke

Klinik für Innere Medizin - Kardiologie,

Schön Klinik Neustadt 